\title{
HNRNPC Promotes Proliferation, Metastasis and Predicts Prognosis in Prostate Cancer
}

\author{
Shiyu Wang ${ }^{1,2}$ \\ Guoxiong Xu (iD ${ }^{3}$ \\ Fan Chao ${ }^{1,2}$ \\ Cong Zhang ${ }^{1,2}$ \\ Dunsheng Han ${ }^{1,2}$ \\ Gang Chen ${ }^{1,2}$ \\ 'Department of Urology, Jinshan \\ Hospital, Fudan University, Shanghai, \\ 201508, People's Republic of China; \\ ${ }^{2}$ Department of Surgery, Shanghai \\ Medical College, Fudan University, \\ Shanghai, 200032, People's Republic of \\ China; ${ }^{3}$ Research Center for Clinical \\ Medicine, Jinshan Hospital, Fudan \\ University, Shanghai, 201508, People's \\ Republic of China
}

\begin{abstract}
Introduction: The incidence of prostate cancer remains high worldwide, while exploring new therapeutic targets for prostate cancer is essential. Heterogeneous nuclear ribonucleoproteins have been proved to regulate tumorigeneses in various cancers. This study aimed to explore the role of HNRNPC in prostate cancer progression.

Methods: HNRNPC expression and its correlation with clinical features and immune infiltration were analyzed by bioinformatics analysis. The effects of HNRNPC on prostate cell proliferation, migration, and invasion were accessed by EdU, colony formation, transwell, and wound-healing assays.
\end{abstract}

Results: The expression level of HNRNPC was significantly increased in prostate cancer tissues and was correlated with the $\mathrm{T}$ stage, $\mathrm{N}$ stage, Gleason score, PSA level, residual tumors, overall survival, disease-specific survival, and progression-free interval of prostate cancer patients. Silencing HNRNPC inhibited the proliferation and metastasis of prostate cancer cells. The expression of HNRNPC was negatively correlated with the infiltration level of most immune cells in prostate cancer. Mechanistically, HNRNPC may function through regulating gene expression at the posttranscriptional level.

Conclusion: HNRNPC could be a potential marker for the treatment and prognosis prediction of prostate cancer.

Keywords: HNRNPC, prostate cancer, proliferation, metastasis, immune infiltration

\section{Plain Language Summary}

The incidence and mortality of prostate cancer keep rising. Studying the factors that affect the occurrence and development of prostate cancer is of great significance for clinical diagnosis, treatment, and prognosis. In this study, we identified a new abnormal expression gene in prostate cancer-HNRNPC and found that its expression level was correlated with multiple clinicopathologic characteristics of prostate cancer, including T stage, $\mathrm{N}$ stage, PSA level, Gleason score, residual tumor, overall survival, disease-specific survival, and progression-free interval. We also found that HNENPC expression level was correlated with immune cells infiltration. Further, we proved that HNRNPC promoted the proliferation, migration, invasion of PCa cells in vitro and predicted its functional mechanism with the help of bioinformatics analysis. Our study may provide a promising target for the diagnostic and therapeutic application in patients with prostate cancer.

\section{Introduction}

Correspondence: Gang Chen Department of Urology, Jinshan Hospital, Fudan University, Shanghai, 201508,

People's Republic of China

Tel +86-2I-34I89990-55I6

Fax +86-2I-67226966

Email chgan365@I26.com
Prostate cancer (PCa) is a dangerous disease in men. In 2021, the estimated cases of $\mathrm{PCa}$ are 248,530 , accounting for $26 \%$ of male tumors and ranking the first in male tumor incidence; the estimated deaths are 34,130, ranking the second in male cancer mortality. ${ }^{1}$ Usually, localized PCa can be treated with radical surgery and achieve 
a good result, but tumor recurrence and metastasis still occur from time to time. Advanced PCa is usually treated with androgen deprivation therapy (ADT). ${ }^{2}$ However, after a period of ADT treatment, most patients will eventually turn into castration-resistant prostate cancer (CRPC), which has relatively been restricted in response to existing treatments. ${ }^{3}$ Once a patient enters a castration-resistant state, he is more likely to die of his PCa than other causes. $^{2}$ Therefore, finding new targets for diagnosis, treatment, and prognostic prediction of $\mathrm{PCa}$ is necessary.

RNA alternative splicing is essential for gene expression regulation and is responsible for various biological processes, such as cellular homeostasis, cellular function regulation, tissue development, and so on. ${ }^{4,5}$ The dysfunction of alternative splicing contributes to many disorders, including cancer. ${ }^{6,7}$ The heterogeneous nuclear ribonucleoprotein (HNRNP) family is the most critical alternative splicing regulator, which complexes with heterogeneous nuclear RNA (hnRNA) and plays essential roles in RNA maturation, stabilization, translocation, and posttranscriptional modification, etc. ${ }^{8-10}$

HNRNPC is the first HNRNP family member found to regulate RNA splicing, ${ }^{11}$ which has two isoforms (C1 and C2) in human. ${ }^{12}$ The up-regulation of HNRNPC expression has been found in various cancers, ${ }^{13-15}$ showing its importance in the occurrence and development of cancer. HNRNPC functions in cancer by regulating the RNA splicing, ${ }^{16}$ RNA expression, ${ }^{17}$ RNA stability, ${ }^{18}$ mRNA translation, ${ }^{19}$ m6A RNA methylation, ${ }^{13,14}$ and dsRNAinduced interferon response. ${ }^{15}$ However, the role of HNRNPC in PCa is still poorly understood.

In the present study, using bioinformatics analysis and molecular biology experiments, we proved the upregulation of HNRNPC in $\mathrm{PCa}$ tissues. The expression level of HNRNPC was correlated with multiple clinicopathologic characteristics and the infiltration level of various immune cells in PCa. The knocking-down of HNRNPC in PCa cell lines effectively inhibited their proliferation, migration, and invasion ability. Our study revealed the vital role of HNRNPC in prostate cancer and provided a potential marker for improving the diagnosis, treatment, and prognosis of prostate cancer.

\section{Materials and Methods}

\section{Patient Tissue Specimens and Cell Lines}

Fifty-two pairs of $\mathrm{PCa}$ and adjacent normal prostate tissues were obtained from PCa patients who underwent radical prostatectomy. All tissues were frozen in liquid nitrogen for storage. All patients agreed that their specimens could be used for scientific research purposes. We have obtained informed consent from all patients for their samples to be used in this study. The privacy rights of all patients were strictly protected. This study was approved by the Ethics Committee of Jinshan Hospital, Fudan University. Human PCa cell lines (PC-3, DU145) and the HEK293T cell line were purchased from FuHeng Cell Center (FuHeng, Shanghai, China). PC-3 was cultured in DME/F12 medium (HyClone, Logan, USA) with 10\% fetal bovine serum (FBS) (BI, Beit Haemek, Israel), HEK293T and DU145 were cultured in DMEM-4.5g/L glucose medium (Gibco, New York, USA) with $10 \%$ FBS. All cell lines were maintained in a humidified incubator containing $5 \% \mathrm{CO} 2$ at $37^{\circ} \mathrm{C}$.

\section{Plasmid Vector Construction and Transfection}

Short hairpin RNAs (shRNAs) targeting HNRNPC mRNA and negative control shRNA were synthesized and cloned into vector PLKO.1 (Genewiz, Suzhou, China). The sequences of shRNAs were listed in Supplementary Table $\underline{\mathrm{S} 1}$. Then, using lipoD293 transfection reagent (SignaGene Laboratories, Rockville, USA), PLKO.1, pSPAX2, and pMD2.G plasmids were co-transfected into HEK293T cells following the manufacturer's protocol. Lentiviral supernatant was collected at 48 and $72 \mathrm{~h}$ after transfection. PC-3 and DU145 cells were seeded in 6-well plates and cultured to $30-50 \%$ confluence at transfection. The lentiviral supernatant was mixed with the complete medium in a $1: 1$ ratio and added to the cells with $10 \mu \mathrm{g} / \mathrm{mL}$ hexadimethrine bromide (Sigma-Aldrich, St. Louis, USA) for 24 hours of infection. Then, 72 hours later, $3 \mu \mathrm{g} / \mathrm{mL}$ puromycin was added into the medium to wipe out non-infected cells.

\section{RNA Extraction and Quantitative Real-Time Polymerase Chain Reaction (qRT-PCR)}

Total RNA of the PCa and normal prostate tissues were extracted using TRIzol Reagent (Takara, Shiga, Japan). RNA-Quick Purification Kit (Yishan, Shanghai, China) was used to extract total RNA from cell lines. RNA purity and concentration were measured using Nanodrop 2000 (Thermo Fisher Scientific, Waltham, USA). Reverse transcriptions were performed using the PrimeScript RT Master Mix (Takara, Shiga, Japan). BeyoFast SYBR Green qPCR Mix (Beyotime, Shanghai, China) was used 
to prepare a $\mathrm{qRT}$-PCR reaction system, and PCR reaction was carried out with ABI 7300 Real-Time PCR system (Thermo Fisher Scientific, Waltham, USA). $\beta$-actin was used as internal control, and all samples were repeated three times. Gene expression was analyzed using the $2^{-\Delta \Delta \mathrm{CT}}$ method. ${ }^{20}$ The sequences of primers were listed in Supplementary Table S2.

\section{Western Blot}

Western blot was conducted as we previously reported. ${ }^{21}$ The antibodies used in this study were listed in Supplementary Table S3.

\section{EdU Assay}

BeyoClick EdU Cell Proliferation Kit with Alexa Fluor 555 (Beyotime, Shanghai, China) was used to further confirm a cell proliferation condition following the manufacturer's protocol. Images were photographed using the Olympus IX73 fluorescence inverted microscope system (Olympus, Tokyo, Japan).

\section{Colony Formation Assay}

Transfected cells were harvested, counted, and seeded in 6-well plates at a density of 2000 cells/well and then cultured for two weeks, then fixed with 4\% PFA for 30 minutes and stained with crystal violet solution for 15 minutes.

\section{Transwell Assay}

For migration assay, an appropriate number of cells were seeded with an FBS-free medium in the upper chamber of the transwell permeable support (Corning, New York City, USA). $500 \mu \mathrm{L}$ medium containing $20 \%$ FBS was filled into the lower chamber. $24 \mathrm{~h}$ (DU145) or $48 \mathrm{~h}$ (PC-3) later, the migrated cells on the bottom side of the upper chamber were fixed using 4\% PFA then stained with 1\% crystal violet solution. Images were photographed using the Olympus IX73 fluorescence inverted microscope system (Olympus, Tokyo, Japan).

For invasion assay, the upper chamber of transwell permeable support was coated with diluted Matrigel Matrix (Corning, New York City, USA) and incubated at $37^{\circ} \mathrm{C}$ for 4 hours before seeding cells. Other steps were the same as the migration assay.

\section{Wound-Healing Assay}

An appropriate number of cells were seeded in 6-well plates and incubated overnight to reach $80 \%$ cell confluence. Then, the cells were scratched with the same $200 \mu \mathrm{L}$ pipette tip and replaced with a serum-free medium. At 0, 24 (PC-3), and 48 (DU145) h after scratching, the images were obtained using the Olympus IX73 fluorescence inverted microscope system (Olympus, Tokyo, Japan).

\section{ONCOMINE Database}

ONCOMINE (https://www.oncomine.org) is a cancer microarray database and web-based data-mining platform aimed at facilitating discovery from genome-wide expression analyses. ${ }^{22}$ Here, we used the ONCOMINE database to access the expression of HNRNPC mRNA in various cancers. The threshold values were set as: p-value: 0.05 , fold change: 1.5 , gene rank: top $10 \%$, data type: mRNA.

\section{The Cancer Genome Atlas (TCGA) Database}

TCGA is a public-funded project that aims to catalog and discover major cancer-causing genomic alterations to create a comprehensive "atlas" of cancer genomic profiles. So far, TCGA researchers have analyzed large cohorts of over 30 human tumors through large-scale genome sequencing and integrated multi-dimensional analyses. ${ }^{23}$ In this study, we downloaded RNA-seq data (FPKM format) of the pancancer dataset (730 normal and 10363 tumors) and PRAD dataset (52 normal and 499 tumors), and clinical information of PRAD dataset from TCGA GDC data portal (https://portal.gdc.cancer.gov/) to analyze the expression level and clinical relevance of HNRNPC.

\section{Gene Expression Omnibus (GEO) Database}

GEO (http://www.ncbi.nlm.nih.gov/geo/) is an international public repository for high-throughput microarray and next-generation sequence functional genomic data sets submitted by the research community. ${ }^{24}$ In the present study, four GEO series (GSE46602, GSE60329, GSE104749, GSE140927) containing localized PCa tumor and normal samples, two GEO series (GSE8511, GSE70770) containing metastasis or castration-resistant PCa, localized PCa, and normal samples were downloaded to analyze the expression level of HNRNPC.

\section{The cBioPortal Database}

The cBioPortal for Cancer Genomics (http://www.cbiopor tal.org/) is an open-access database for the exploration of 


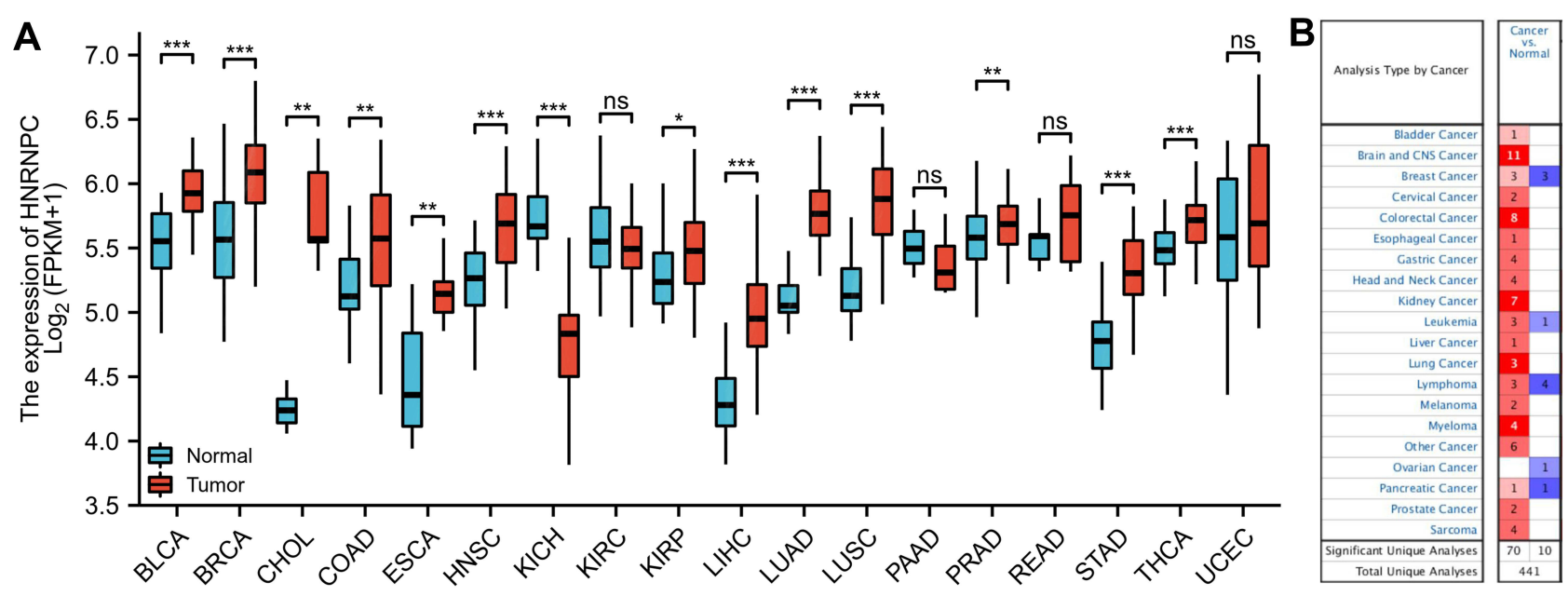

Figure I The expression of HNRNPC mRNA in various types of cancers. (A) TCGA database (paired samples), Wilcoxon signed-rank test. (B) ONCOMINE database, the threshold values were set as following: $\mathrm{P}$-value: 0.05 , fold change: 1.5 , gene rank: top $10 \%$, data type: mRNA. The graphic represents the numbers of datasets in which HNRNPC mRNA expressions were up-regulated (red) or down-regulated (blue). ${ }^{*} \mathrm{p}<0.05,{ }^{* *} \mathrm{p}<0.0 \mathrm{I},{ }^{* * *} \mathrm{p}<0.00 \mathrm{I}$.

multi-dimensional cancer genomics datasets. ${ }^{25}$ We downloaded the "mRNA expression (FPKM polyA)" data of the SU2C dataset ${ }^{26}$ with the help of the cBioPortal database and conducted immune infiltration analysis using the single sample GSEA (ssGSEA) method (a built-in algorithm of R package GSVA). ${ }^{27-29}$ The gene sets that define each type of immune cell were reported by Bindea et al. ${ }^{30}$

\section{Metascape}

Metascape (https://metascape.org) is a web-based portal that provides a comprehensive gene list annotation and analysis resource for experimental biologists. It combines functional enrichment, interactome analysis, gene annotation, and membership search to leverage over 40 independent knowledgebases within one integrated portal. ${ }^{31}$ Here, we used the $\mathrm{R}$ package stat to search the positive co-expression genes of HNRNPC based on the data from TCGA PRAD. The top 50 (ranked by spearman coefficient) co-expression genes with HNRNPC itself were uploaded into Metascape. Then, the "express analysis" function was used to achieve gene enrichment analysis and co-expression network analysis.

\section{Statistical Analysis}

All experiments were conducted at least three times. Statistical analyses were performed using $\mathrm{R}$ (v.3.6.3) or GraphPad Prism (v.8.0.2). According to different data types, appropriate statistical methods such as Student's $t$-test, Welch's $t$-test, One-way ANOVA test, Wilcoxon matchedpairs signed-rank test, Wilcoxon rank-sum test, chi-squared test were used for comparisons. The significant levels were set at: ${ }^{*} p<0.05, * * p<0.01,{ }^{* * *} p<0.001$ and ${ }^{* * * *} p<0.0001$.

\section{Results}

\section{HNRNPC Expression Comparison}

Pan-cancer analysis was conducted using TCGA and ONCOMINE database. A total of 18 types of cancer in the TCGA database were analyzed (Figure 1A), the expression of HNRNPC was significantly up-regulated in 13 cancers, including bladder urothelial carcinoma, breast invasive carcinoma, cholangiocarcinoma, colon adenocarcinoma, esophageal carcinoma, head and neck squamous cell carcinoma, kidney renal papillary cell carcinoma, liver hepatocellular carcinoma, lung adenocarcinoma, lung squamous cell carcinoma, prostate adenocarcinoma, stomach adenocarcinoma, and thyroid

Table I Details of GEO Series Included in This Analysis

\begin{tabular}{|l|l|l|}
\hline $\begin{array}{l}\text { GEO } \\
\text { Series }\end{array}$ & Contributors & Platform \\
\hline GSE85II & $\begin{array}{l}\text { Poisson LM } \\
\text { et al, 20II }\end{array}$ & $\begin{array}{l}\text { Agilent-0I239I Whole Human } \\
\text { Genome Oligo Microarray G4I I2A }\end{array}$ \\
\hline GSE46602 & $\begin{array}{l}\text { Mortensen } \\
\text { MM et al, 20I5 }\end{array}$ & $\begin{array}{l}\text { Affymetrix Human Genome UI33 } \\
\text { Plus 2.0 Array }\end{array}$ \\
\hline GSE60329 & $\begin{array}{l}\text { Chiorino } \\
\text { G et al, 20I7 }\end{array}$ & $\begin{array}{l}\text { Agilent-028004 SurePrint G3 } \\
\text { Human GE 8x60K Microarray }\end{array}$ \\
\hline GSE70770 & $\begin{array}{l}\text { Ross-Adams } \\
\text { H et al, 20I5 }\end{array}$ & $\begin{array}{l}\text { Illumina HumanHT-I2 V4.0 } \\
\text { expression beadchip }\end{array}$ \\
\hline GSE104749 & $\begin{array}{l}\text { Shan M et al, } \\
2017\end{array}$ & $\begin{array}{l}\text { Affymetrix Human Genome UI33 } \\
\text { Plus 2.0 Array }\end{array}$ \\
\hline GSEI40927 & $\begin{array}{l}\text { Wang J et al, } \\
2019\end{array}$ & $\begin{array}{l}\text { Agilent-078298 human ceRNA array } \\
\text { VI.0 4XI80K }\end{array}$ \\
\hline
\end{tabular}


carcinoma. In ONCOMINE analysis (Figure 1B), HNRNPC was found to be significantly over-expressed in 70 datasets among 19 out of 20 cancer types, while down-expressed in only ten datasets. In PCa, HNRNPC over-expression was found in 2 datasets, no down-expression dataset was found.

To further verify the expression of HNRNPC in PCa, six GEO series were analyzed. Detailed information on the included GEO series is listed in Table 1. Compared with normal samples, HNRNPC was significantly up-regulated in PCa samples (Figure 2A-F). Moreover, in metastasis PCa or CRPC samples, the expression of HNRNPC was even higher than in localized PCa (Figure 2E-F). Besides, unpaired and paired samples of TCGA also suggested the over-expression of HNRNPC in PCa (Figure $2 \mathrm{G}$ and $\mathrm{H}$ ). Further, we collected $\mathrm{PCa}$ and adjacent tissues from radical prostatectomy specimens and extracted RNA from them. The following qRT-PCR results also confirmed the overexpression of HNRNPC in PCa (Figure 2I).
A

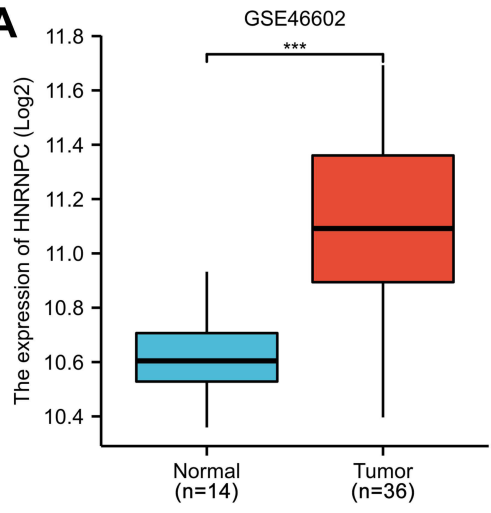

D

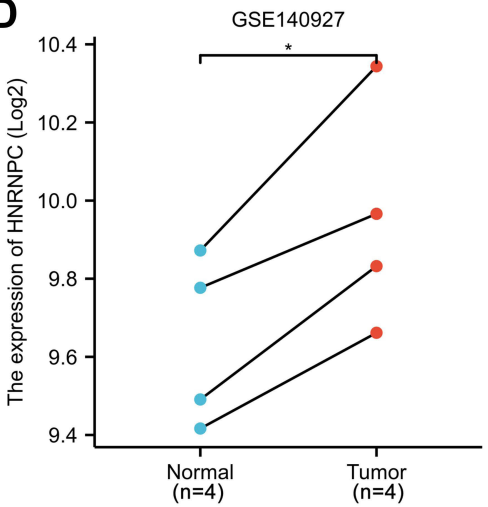

G

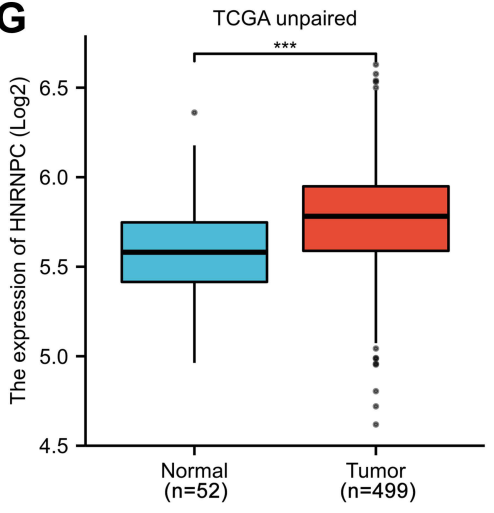

B

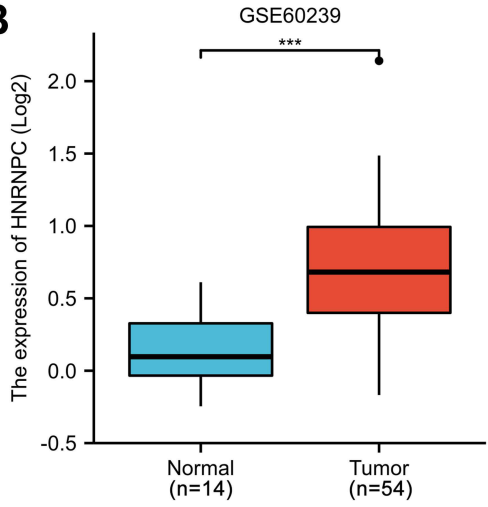

E

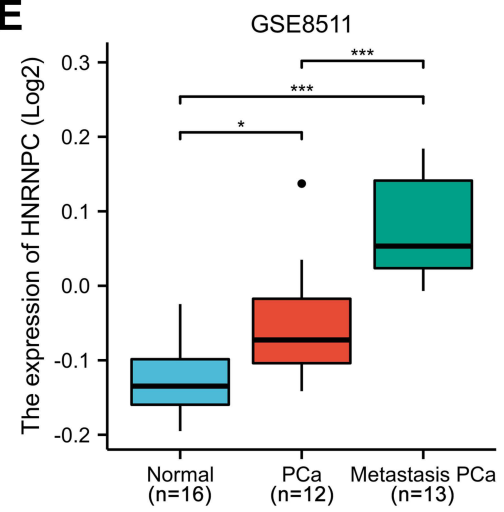

H

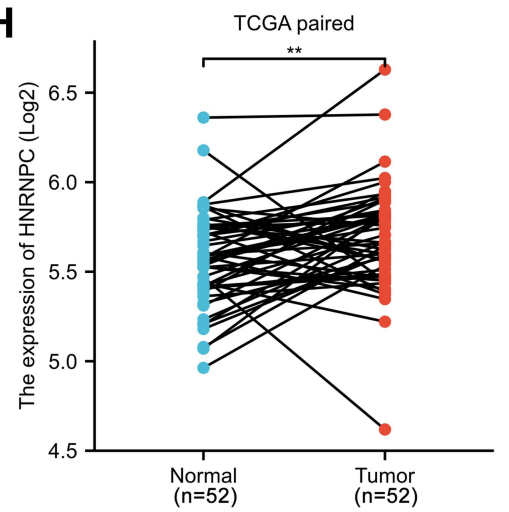

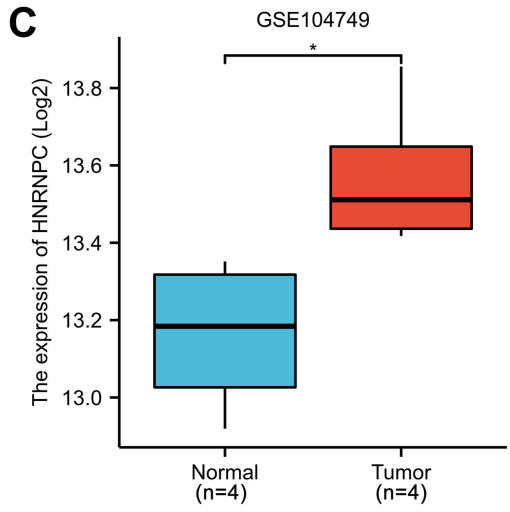

$\mathbf{F}$

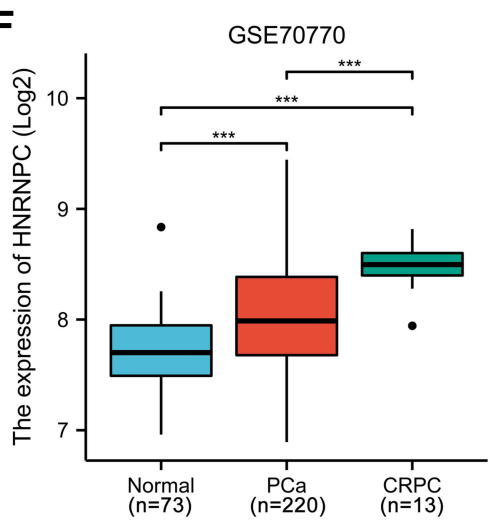

I

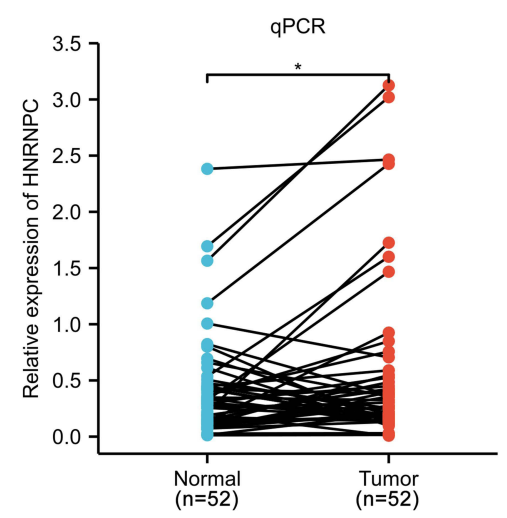

Figure 2 The expression of HNRNPC mRNA in PCa tissues and normal prostate tissues (GEO and TCGA database). (A) GSE46602, Welch's t-test. (B) GSE60239, students' t-test. (C) GSEI 04749, students' $t$-test. (D) GSEI40927, students' $t$-test (paired). (E) GSE85I I, One-way ANOVA, and Bonferroni's multiple comparisons test. (F) GSE70770, One-way ANOVA, and Bonferroni's multiple comparisons test. (G) TCGA PRAD, unpaired samples, Wilcoxon rank-sum test. (H) TCGA PRAD, paired samples, Wilcoxon signed-rank test. (I) qPCR verification of PCa samples collected by ourselves, paired samples, Wilcoxon signed-rank test. ${ }^{*} \mathrm{p}<0.05$, ${ }^{* *} \mathrm{p}<0.0 \mathrm{I}$, $* * * \mathrm{p}<0.00 \mathrm{I}$. 
Table 2 Correlation Between HNRNPC Expression and Clinicopathological Characteristics in PCa

\begin{tabular}{|c|c|c|c|}
\hline Characteristic & $\begin{array}{l}\text { Low Expression } \\
\text { of HNRNPC }\end{array}$ & $\begin{array}{c}\text { High Expression } \\
\text { of HNRNPC }\end{array}$ & $\mathbf{p}$ \\
\hline $\begin{array}{l}\text { Age, n (\%) } \\
\quad \leq 60 \\
>60\end{array}$ & $\begin{array}{l}\text { II (22.2\%) } \\
\text { I } 38 \text { (27.7\%) }\end{array}$ & $\begin{array}{l}\text { II } 3 \text { (22.6\%) } \\
\text { I37 (27.5\%) }\end{array}$ & 0.960 \\
\hline $\begin{array}{l}\text { T stage, } \mathrm{n}(\%) \\
\text { T2 } \\
\text { T3\&T4 }\end{array}$ & $\begin{array}{l}\text { IIO (22.4\%) } \\
\text { I34 (27.2\%) }\end{array}$ & $\begin{array}{c}79(16.1 \%) \\
169(34.3 \%)\end{array}$ & 0.003 \\
\hline $\begin{array}{l}\mathrm{N} \text { stage, } \mathrm{n}(\%) \\
\text { No } \\
\text { NI }\end{array}$ & $\begin{array}{c}177(41.5 \%) \\
29(6.8 \%)\end{array}$ & $\begin{array}{c}170(39.9 \%) \\
50(11.7 \%)\end{array}$ & 0.030 \\
\hline $\begin{array}{l}\text { M stage, n (\%) } \\
\text { M0 } \\
\text { MI }\end{array}$ & $\begin{array}{c}225(49.1 \%) \\
\mathrm{I}(0.2 \%)\end{array}$ & $\begin{array}{c}230(50.2 \%) \\
2(0.4 \%)\end{array}$ & 1.000 \\
\hline $\begin{array}{l}\text { PSA }(\mathrm{ng} / \mathrm{mL}) \text {, } \\
\mathrm{n}(\%) \\
\quad<4 \\
\quad \geq 4\end{array}$ & $\begin{array}{c}215(48.6 \%) \\
10(2.3 \%)\end{array}$ & $\begin{array}{c}200(45.2 \%) \\
17(3.8 \%)\end{array}$ & 0.197 \\
\hline $\begin{array}{l}\text { Gleason score, } \\
\text { n (\%) } \\
\quad 6 \& 7 \\
\quad 8,9 \& 10\end{array}$ & $\begin{array}{c}173(34.7 \%) \\
76(15.2 \%)\end{array}$ & $\begin{array}{l}120(24 \%) \\
130(26.1 \%)\end{array}$ & $\begin{array}{c}< \\
0.001\end{array}$ \\
\hline
\end{tabular}

Notes: All comparisons were conducted using chi-squared test. Bold text indicates statistically significant $p$-values.

\section{HNRNPC Expression is Correlated with Poor Clinicopathological Features and Prognosis of $\mathrm{PCa}$}

Four hundred and ninety-nine PCa patients of the TCGA PRAD dataset were divided into the HNRNPC low expression group $(\mathrm{n}=249)$ and HNRNPC high expression group $(n=250)$ by the median. The clinicopathological features are shown in Table 2. HNRNPC expression was significantly correlated with the $\mathrm{T}$ stage $(p<0.001), \mathrm{N}$ stage $(p=0.03)$, and Gleason score $(p<0.001)$.

The logistic regression method was also used to show the relationship between the clinicopathological characteristics of PCa and the expression level of HNRNPC. The results also suggested that HNRNPC was significantly related to the $\mathrm{T}$ stage, $\mathrm{N}$ stage, and Gleason score (Supplementary Table S4).

When grouped by clinicopathological features, HNRNPC expression was significantly correlated with the $\mathrm{T}$ stage (Figure $3 \mathrm{~A}$ ), the $\mathrm{N}$ stage (Figure 3B), the Gleason score (Figure 3C), the PSA level (Figure 3D), and the residual tumor (Figure 3E). In addition, the receiver operating characteristic (ROC) curve was used to analyze the effectiveness of HNRNPC expression level to distinguish PCa tissues from normal prostate tissues. The area under the curve (AUC) of HNRNPC was 0.710 (Figure 3F), suggesting that HNRNPC could be served as a biomarker to distinguish $\mathrm{PCa}$ from normal prostate tissue. Besides, the Kaplan-Meier method was also used to evaluate the association between HNRNPC expression and patients' survival. The results showed that the expression of HNRNPC is significantly correlated with poor OS, DSS, and PFI of PCa patients (Figure 3G-I).

\section{HNRNPC Promotes PCa Cells Proliferation and Metastasis in vitro}

HNRNPC specific shRNAs were constructed into plasmids and transfected into PCa cell lines. The expression of HNRNPC mRNA and protein was significantly knocked down in both PC-3 and DU145 cells (Figure 4A-C).

First of all, the colony formation assay showed that the colony numbers and sizes were significantly reduced when HNRNPC was silenced (Figure 4D). Then, an EdU assay, which is the gold standard for testing cell proliferation ability, was conducted. The results showed that the loss of function of HNRNPC significantly reduced the percentages of EdU-positive cells in PC-3 and DU145 cells (Figure 4E-H). The above assays confirmed that HNRNPC plays a positive role in $\mathrm{PCa}$ cell proliferation.

A transwell assay was firstly performed to explore the effect of HNRNPC on PCa cell metastasis. The results proved that the knocking-down of HNRNPC significantly suppressed the migration and invasion of PC-3 and DU145 cells (Figure 4I and J). However, the result of the transwell assay was easily affected by cell proliferation. So, we supplemented the wound-healing assay, in which cells were cultured in a serum-free medium to exclude the influence of cell proliferation. The result confirmed that the loss of function of HNRNPC inhibited migration in PC-3 and DU145 cells (Figure $4 \mathrm{~K}$ and $\mathrm{J}$ ). 
A

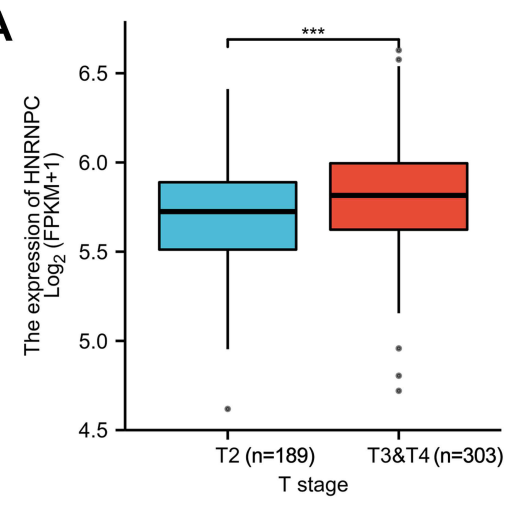

D
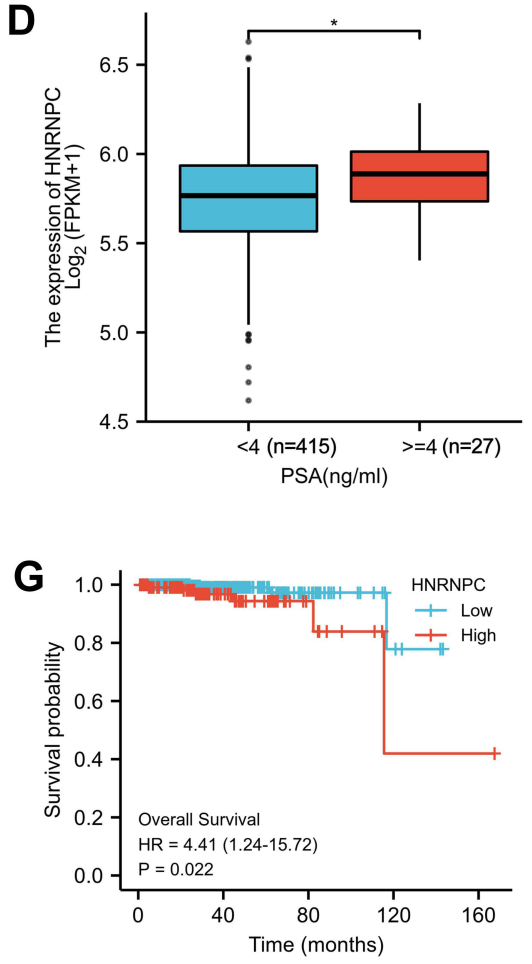

B

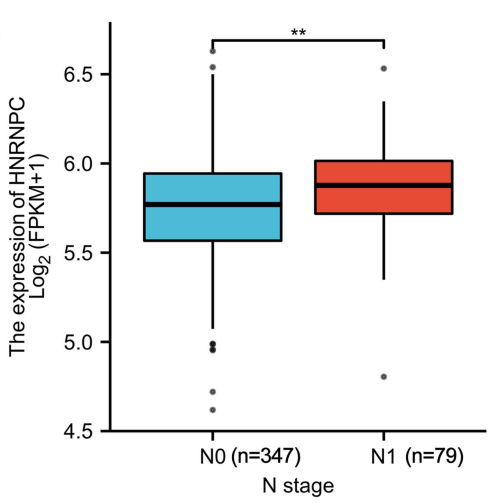

E

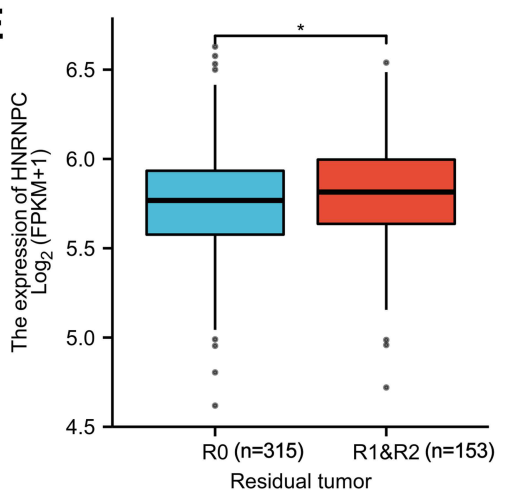

H

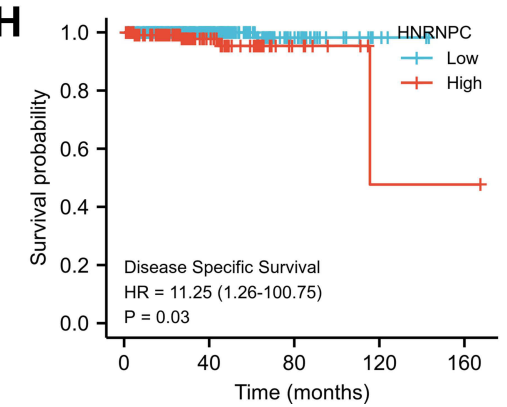

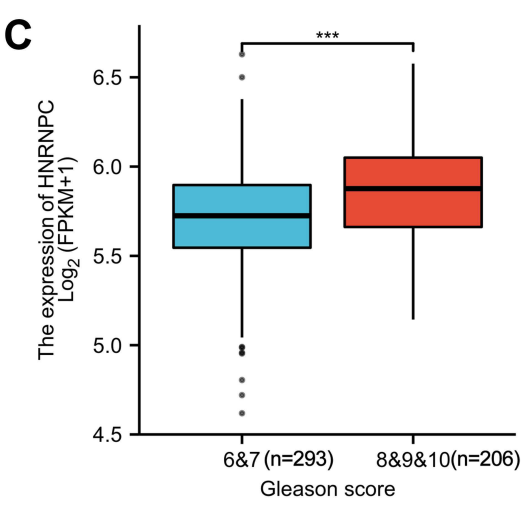

F
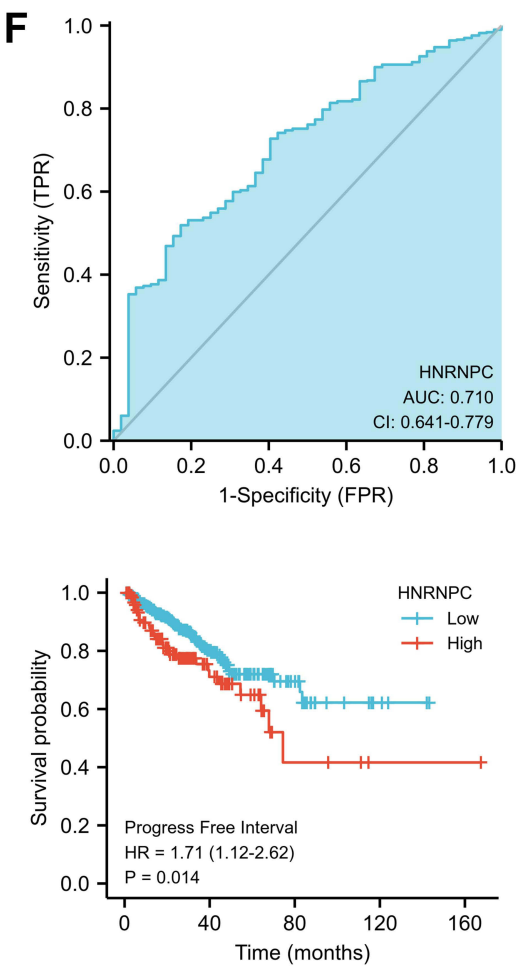

Figure 3 Relationship between HNRNPC mRNA expression and clinicopathologic characteristics in PCa (TCGA database). (A) The relationship between HNRNPC mRNA expression and T stage, Wilcoxon rank-sum test. (B) The relationship between HNRNPC mRNA expression and N stage, Wilcoxon rank-sum test. (C) The relationship between HNRNPC mRNA expression and Gleason score, Wilcoxon rank-sum test. (D) The relationship between HNRNPC mRNA expression and PSA level, Wilcoxon rank-sum test. (E) The relationship between HNRNPC mRNA expression and Residual tumor, Wilcoxon rank-sum test. (F) ROC curve showed the efficiency of HNRNPC mRNA expression to distinguishing PCa tissue from non-tumor tissue. (G-I) Kaplan-Meier curve showed the prognostic value of HNRNPC in OS, DSS, and PFI of PCa patients. ${ }^{*} \mathrm{p}<0.05,{ }^{* *} \mathrm{p}<0.01$, *** $\mathrm{p}<0.001$.

\section{HNRNPC Expression is Correlated with Immune Infiltration in $\mathrm{PCa}$}

We further analyzed the correlation between HNRNPC expression and immune infiltration. The results showed that HNRNPC expression was negatively correlated with most immune cells' infiltration levels (Figure 5A, Supplementary Table S5). Among them, it has the strongest correlation with natural killer (NK) cells, effector memory $\mathrm{T}(\mathrm{Tem})$ cells, and plasmacytoid dendritic cells
(pDCs) (Figure 5B-D, F-H). On the other hand, HNRNPC expression was positively correlated with $\mathrm{T}$ helper 2 (Th2) cells (Figure 5E and I).

\section{Predicted HNRNPC Related Functions and Pathways in PCa}

The heatmap of the top 50 co-expressed genes with HNRNPC is shown in Figure 6. Enrichment analysis of HNRNPC and its top 50 co-expression genes was 

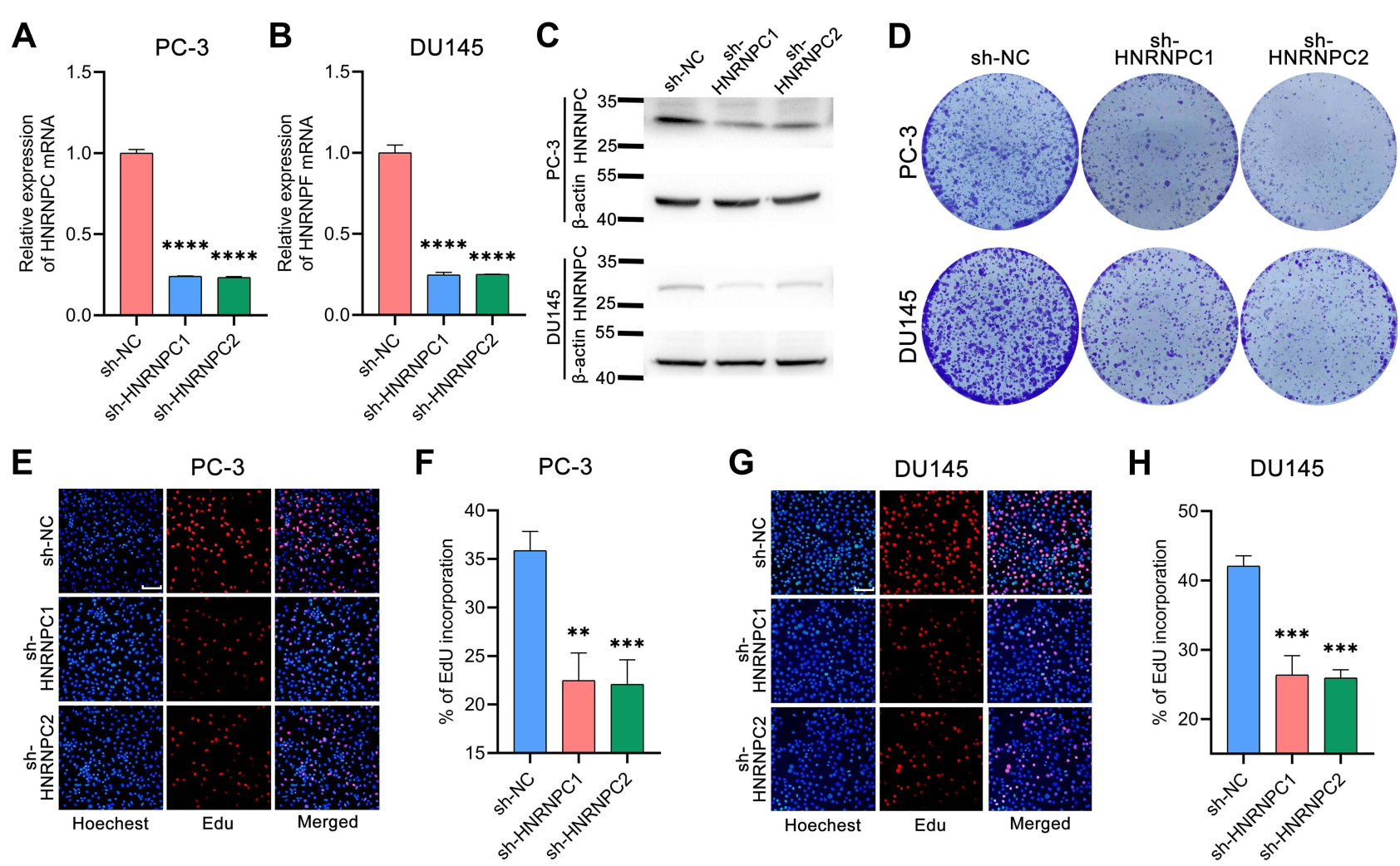

I

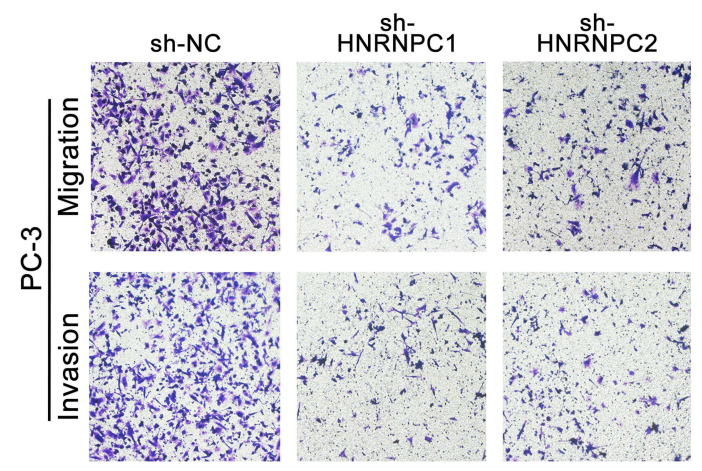

J
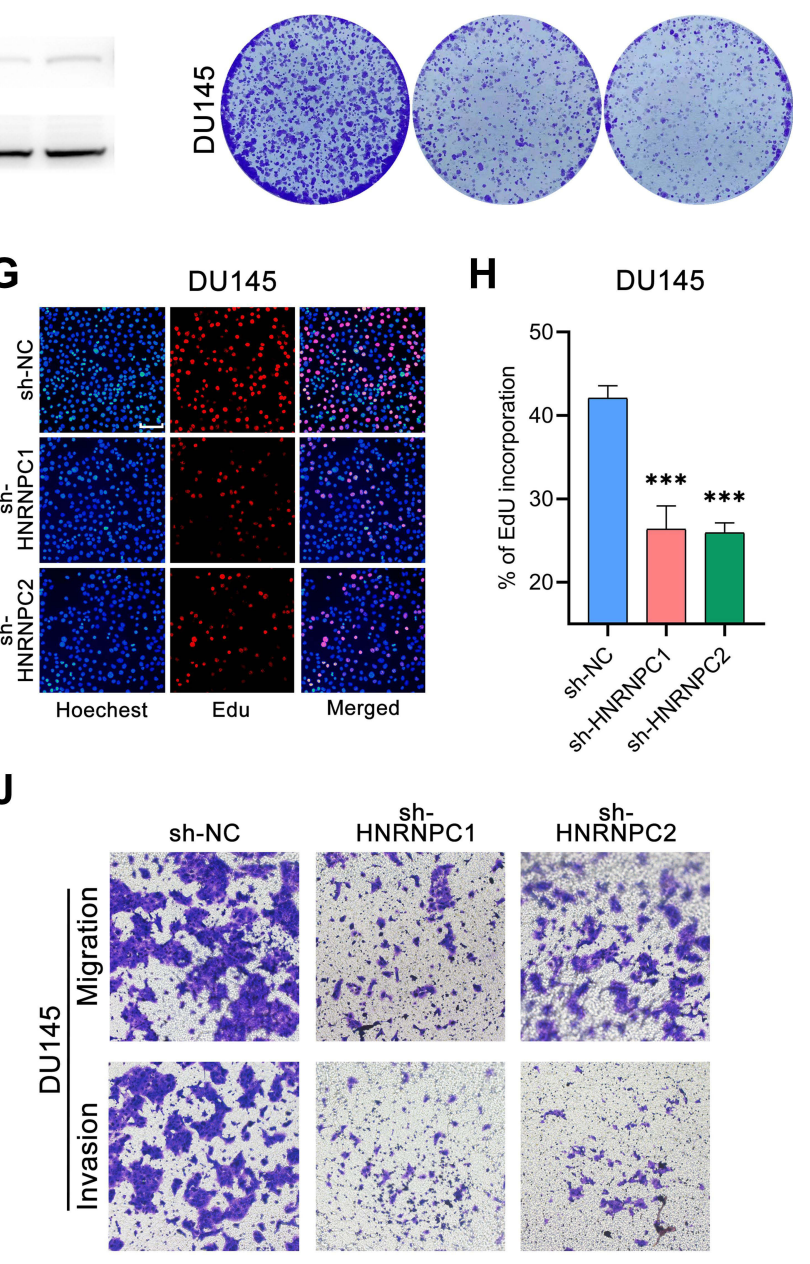

K

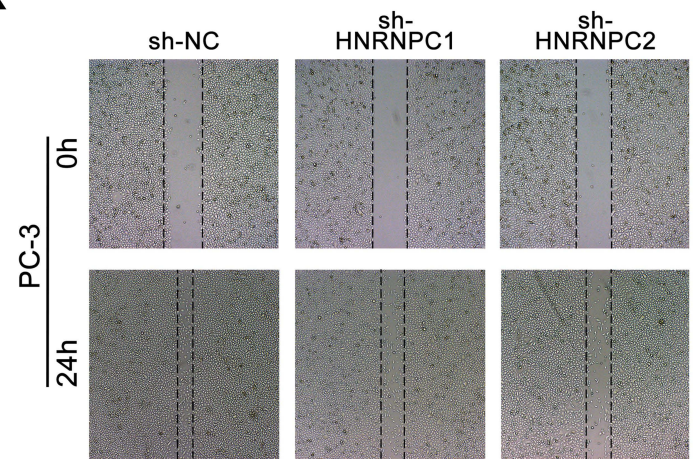

L

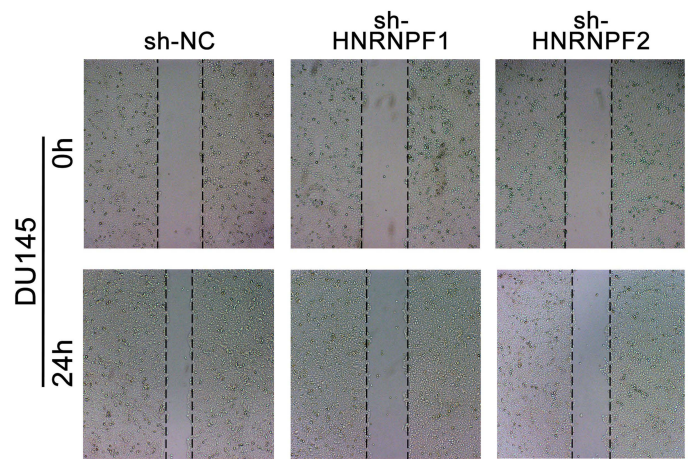

Figure 4 Knocking-down HNRNPC inhabits PCa cell proliferation, migration, and invasion in vitro. (A and B) qRT-PCR of the relative expression of HNRNPC mRNA in PC-3 and DUI 45 cells transfected with HNRNPC specific shRNA plasmids or negative control shRNA plasmid. The data are presented as the mean \pm SD. One-way ANOVA and Dunnett's multiple comparisons test, $n=3$. (C) Western blot of the relative expression of HNRNPC protein in PC-3 and DUI45 cells transfected with HNRNPC specific shRNA plasmids or negative control shRNA plasmid. (D) Colony formation assays of the above-mentioned different transfected PCa cells. (E-H) EdU assays and their corresponding column charts of different transfected PCa cells. Scale bar, 100um. The data are presented as the mean \pm SD. One-way ANOVA and Dunnett's multiple comparisons test, $\mathrm{n}=3$. ( $\mathbf{I}$ and $\mathbf{J})$ Transwell assay of different transfected PCa cells. ( $\mathbf{K}$ and $\mathbf{L}$ ) Wound-healing assay of different transfected PCa cells. $* * p^{\circ}<0.0 \mathrm{I}$, *** $\mathrm{p}<0.00 \mathrm{I}$, $* * * * 0<0.0001$. 


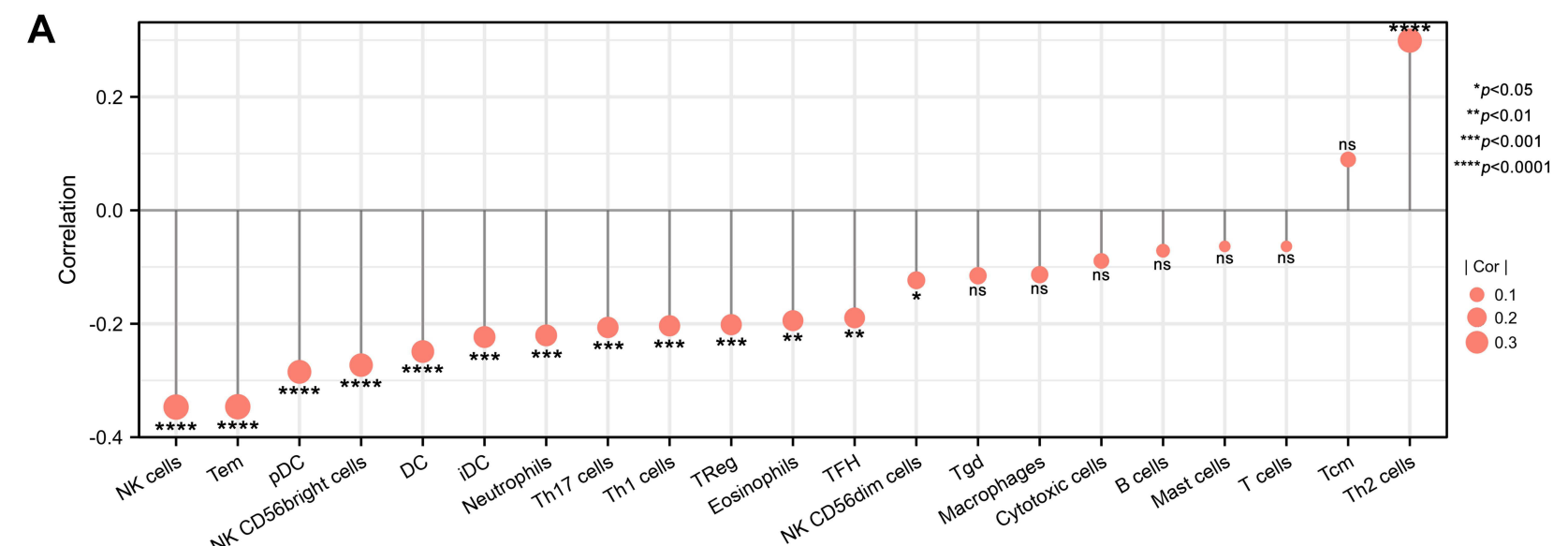

B

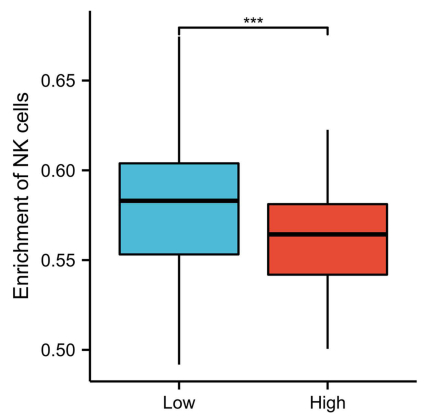

$\mathbf{F}$

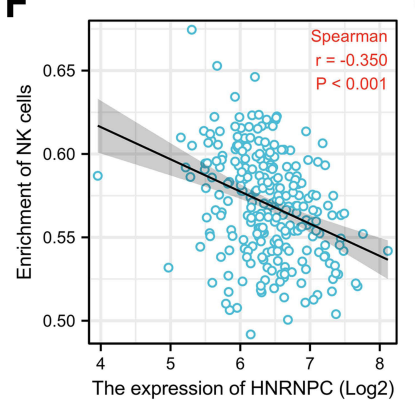

C

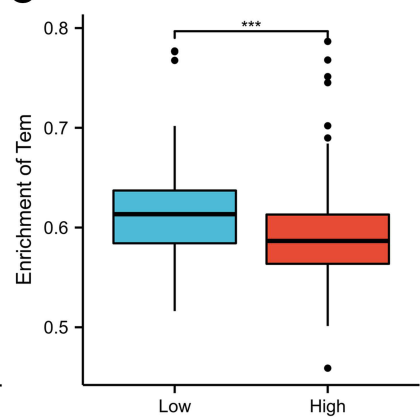

G

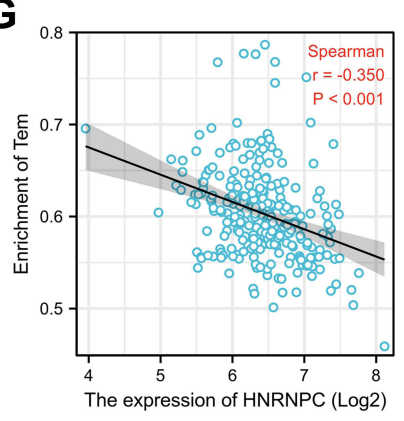

D

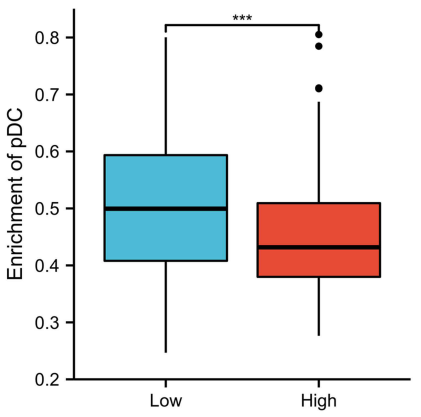

H

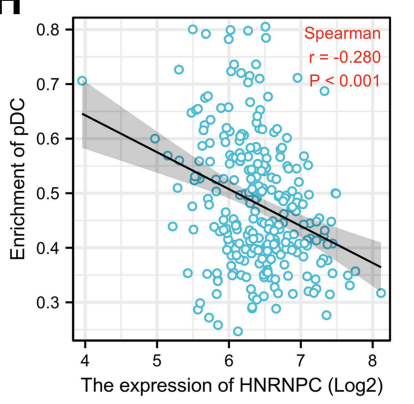

$\mathbf{E}$

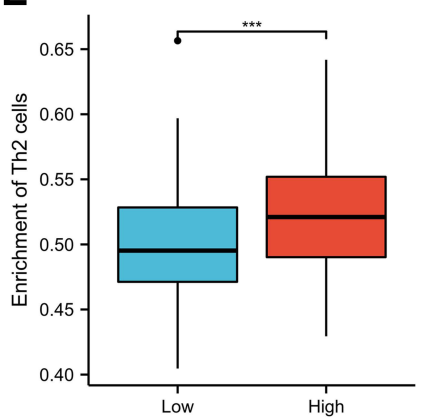

I

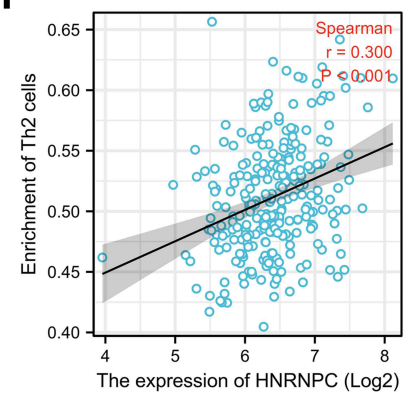

Figure 5 Relationship between HNRNPC mRNA expression and immune infiltration in the tumor microenvironment. (A) The forest plot of the correlation between HNRNPC mRNA expression and various immune cells. (B-E) The infiltration level of NK cells, Tem, pDC, and Th2 cells in HNRNPC high and low expression groups. Wilcoxon rank-sum test, $n=133$. (F-I) The correlation between HNRNPC mRNA expression and the infiltration level of NK cells, Tem, $p D C$, and Th2 cells. ${ }^{*} p<0.05$, $* * p<0.01, * * * p<0.001$, **** $p<0.0001$.

conducted using Metascape. The significantly enriched terms are listed in Figure 7A. Besides, the enriched pathway could interact and form a network (Figure 7B).

\section{Discussion}

The HNRNP family is a large protein family with more than 20 members (HNRNP A-U), which regulates alternative splicing and affects the maturation, stabilization, translocation, and translation of mRNAs. ${ }^{32}$ HNRNPs were also reported to participate in the formation of lncRNAs and circRNAs. ${ }^{33,34}$ For example, HNRNPP2 (FUS) is a famous non-coding RNA regulator. ${ }^{35}$ Many HNRNPs have been reported to play important roles in tumorigenesis. For example, HNRNPA1 promotes tumor proliferation in lung cancer and tumor metastasis in breast cancer; ${ }^{36,37}$ HNRNPA2/B1 affects the proliferation of various tumor cell lines; ${ }^{38}$ HNRNPL was reported as a regulator in prostate cancer. ${ }^{34}$ HNRNPF promotes tumor progression in colorectal cancer, ${ }^{39}$ bladder cancer, ${ }^{40,41}$ and thyroid cancer. ${ }^{42}$

HNRNPC was one of the first identified members of the HNRNP family and was previously studied in various malignant tumors. ${ }^{13-15}$ In glioblastoma, Wang et $\mathrm{al}^{13}$ reported that HNRNPC was up-regulated in glioma tissues compared with normal brain tissues, and higher HNRNPC expression level related to higher clinical stage. Park et al ${ }^{17}$ 


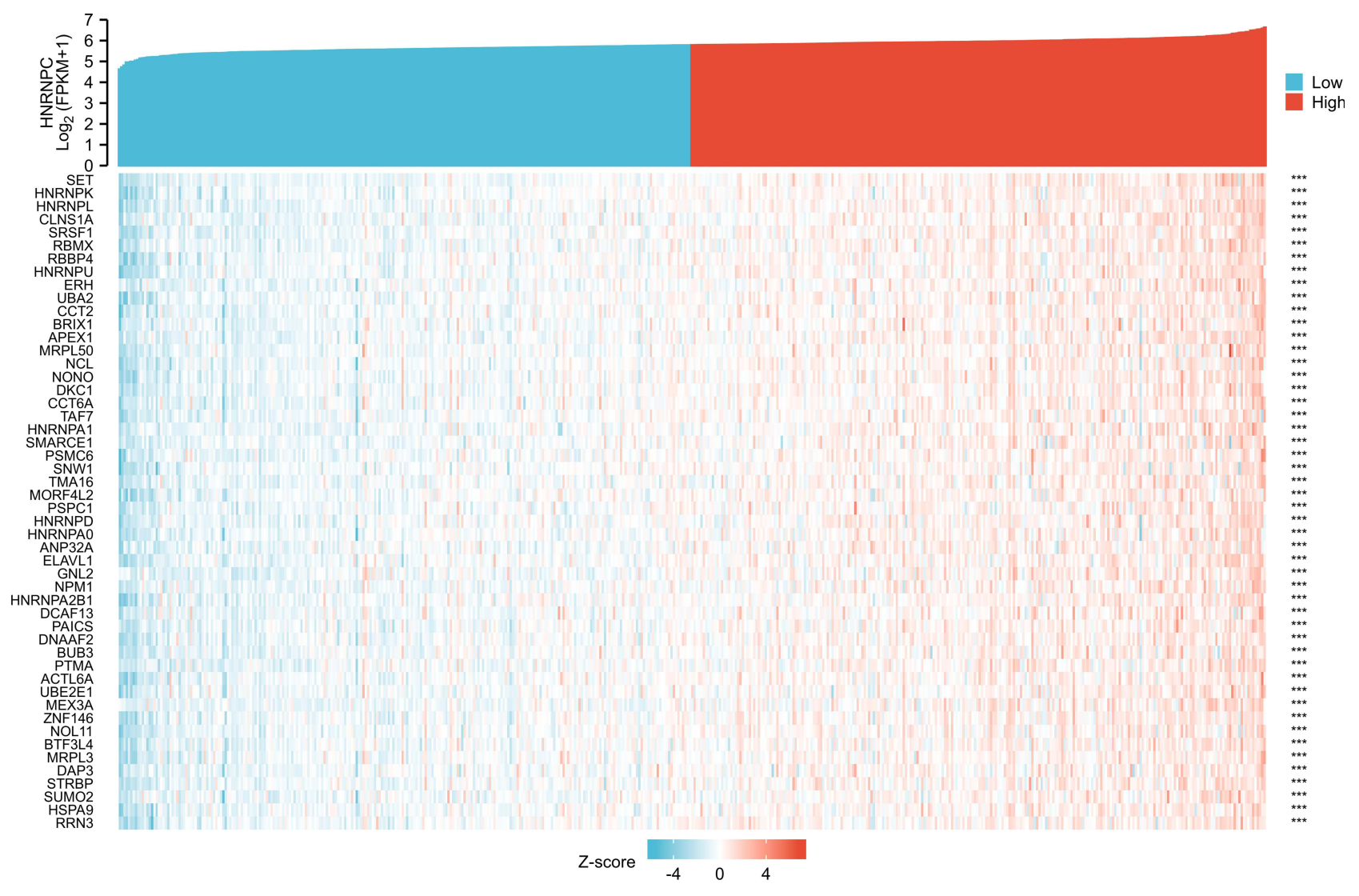

Figure 6 Heat map of top 50 co-expressed genes with HNRNPC (TCGA database).

further proved that silencing of HNRNPC in glioblastoma cell lines inhibited their proliferation and metastasis abilities. Besides, Huang et $\mathrm{al}^{14}$ reported that HNRNPC was overexpressed in oral squamous cell carcinoma (OSCC), and the higher HNRNPC expression level was positively correlated with advanced clinical stage, lymph node metastasis, and undesirable overall survival. Besides, they also proved that HNRNPC promotes proliferation, migration, and invasion of OSCC cell lines. Similar results were also reported by $\mathrm{Wu}$ et $\mathrm{al}^{15}$ in breast cancer.

However, the role of HNRNPC in prostate cancer has not been well studied. In this study, we found that HNRNPC mRNA expression was up-regulated in PCa tissues. Then, we explored the relationship between HNRNPC mRNA expression level and clinicopathological features in PC and found that higher HNRNPC expression level was related to higher $\mathrm{T}$ stage, $\mathrm{N}$ stage, Gleason score, PSA level, residual tumor event, and worse survival prognosis. Then, using PCa cell lines, we proved that silencing HNRNPC inhibited cell proliferation, migration, and invasion in PCa. The results we obtained through bioinformatics analysis and cell experiments are consistent with the results reported by previous studies on other malignant tumors, which indicates that HNRNPC may be an oncogene and plays an essential role in the occurrence and development of PCa.

Alternative splicing of RNA, such as retention of introns, skipping of exons, mutually exclusive exons, change of alternative splice sites, is a common biological process. $^{43,44}$ Aberrant alternative splicing has been reported to play an important role in various physiological and pathological processes, including aging, ${ }^{45}$ angiogenesis, ${ }^{46}$ spermatogenesis, ${ }^{47}$ cardiovascular disease, ${ }^{48}$ diabetes, ${ }^{43}$ neurological diseases, ${ }^{49}$ and cancer. ${ }^{6,7}$ Hypoxia-induced alternative splicing was even reported as the 11 th hallmark of cancer. ${ }^{50}$ As a vital alternative splicing regulator, HNRNPC is essential for the normal flow of genetic information. For example, Fischl et $\mathrm{al}^{16}$ reported that overexpression of HNRNPC has a critical role in the alternative cleavage and polyadenylation of metastatic colon cancer cells. Park et $\mathrm{al}^{17}$ found that the silencing of HNRNPC decreases the level of miR21, then increases the expression of PDCD4 and PTEN, and inhibits the metastatic potential of the glioblastoma. 
A

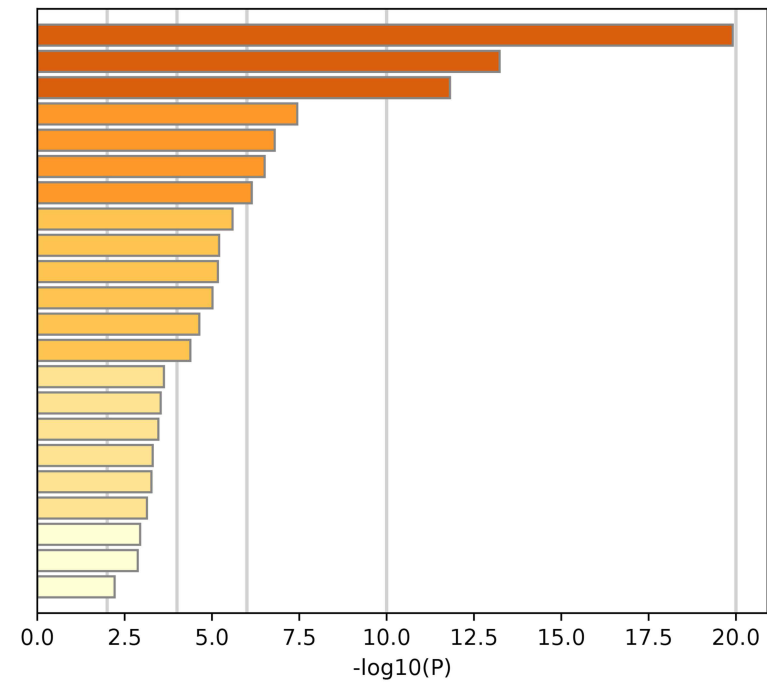

GO:1903311: regulation of mRNA metabolic process GO:0043487: regulation of RNA stability

GO:0032204: regulation of telomere maintenance

CORUM:778: LARC complex (LCR-associated remodeling complex)

GO:0022613: ribonucleoprotein complex biogenesis

CORUM:3135: TLE1 corepressor complex (MASH1 promoter-corepressor complex)

GO:0071824: protein-DNA complex subunit organization

GO:0006913: nucleocytoplasmic transport

GO:0032211: negative regulation of telomere maintenance via telomerase

GO:0018205: peptidyl-lysine modification

GO:0048026: positive regulation of mRNA splicing, via spliceosome

R-HSA-2990846: SUMOylation

GO:0070897: transcription preinitiation complex assembly

R-HSA-1640170: Cell Cycle

GO:0006412: translation

GO:0032984: protein-containing complex disassembly

GO:0071407: cellular response to organic cyclic compound

WP3594: Circadian rhythm related genes

GO:0022618: ribonucleoprotein complex assembly

GO:2000736: regulation of stem cell differentiation

GO:0072331: signal transduction by p53 class mediator

GO:0040029: regulation of gene expression, epigenetic

B

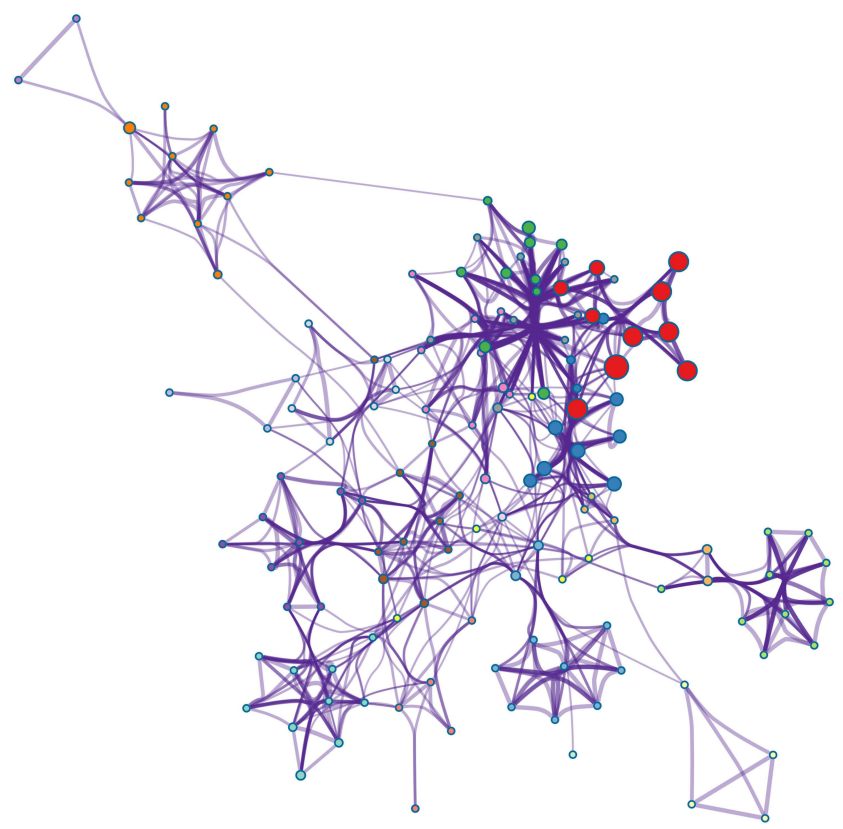

regulation of mRNA metabolic process

regulation of RNA stability

regulation of telomere maintenance

LARC complex

ribonucleoprotein complex biogenesis

TLE1 corepressor complex

protein-DNA complex subunit organization

nucleocytoplasmic transport

negative regulation of telomere maintenance

peptidyl-lysine modification

positive regulation of mRNA splicing

sumOylation

transcription preinitiation complex assembly

Cell Cycle

translation

protein-containing complex disassembly

cellular response to organic cyclic compound

Circadian rhythm related genes

ribonucleoprotein complex assembly

regulation of stem cell differentiation

Figure 7 Predicted pathways of HNRNPC and its top 50 co-expression genes (Metascape). (A) Top 22 enriched pathways. (B) Interaction network of enriched pathways.

Shetty ${ }^{18}$ reported that HNRNPC bound to the $3^{\prime} \mathrm{UTR}$ region of uPAR mRNA and stabilized it. Meng et al found that HNRNPC enhances the IRES-mediated translation initiation of IGF-IR.

The mechanism of HNRNPC in PCa was still not clear. We believe that genes with similar expression patterns as HNRNPC are more likely to exist in the same pathway and perform the same function as HNRNPC. Therefore, we conducted gene co-expression and pathway enrichment analysis. HNRNPC and its top 50 co-expression genes were mostly enriched in pathways that regulate the RNA metabolism, splicing, stability, localization, and translation, which can significantly impair the normal cell physical activities, such as the cell cycle. It is worth noting that the well-known tumor-related pathways p53 signaling pathway was also enriched. However, the specific mechanism needs further experimental verification.

In immune infiltration analysis, we found that HNRNPC expression was negatively correlated with the infiltration of most immune cells, especially NK cells, Tem and pDC. NK cells can kill cancer cells directly and enhance antibody and T cell responses to fight cancers. ${ }^{51,52}$ Impaired NK cells or NK cell deficiency have been associated with an increased incidence of various types of cancer. ${ }^{53}$ PDCs can detect 
pathogen-derived nucleic acids and produce type I interferon (IFN-I) rapidly and massively, promoting anti-viral and antitumor immunity. ${ }^{54}$ The dysfunction of $\mathrm{pDCs}$ and exhaustion of IFN-I have been implicated in contributing to the pathogenesis of various cancers. ${ }^{55,56}$ Tcm cells have long-term memory ability and can be home to lymph nodes after antigens activate the original $\mathrm{T}$ cells. Under the restimulation of antigens, Tcm cells can produce a large number of effect memory $\mathrm{T}$ (Tem) cells with immediate effects. ${ }^{57} \mathrm{NK}$ cells, Tem, and pDC are anti-tumor immune cells. Their negative correlation with HNRNPC indicates that immunological mechanisms may be involved in the protumorigenic effect of HNRNPC.

In addition, the expression level of HNRNPC was positively correlated with $\mathrm{Th} 2$ cell infiltration and negatively correlated with Th1 cell infiltration. Th1 cells drive the type 1 pathway (cellular immunity) to fight viruses and other intracellular pathogens and destroy cancer cells. Th2 cells drive the type 2 pathway (humoral immunity) and up-regulate antibody production against extracellular organisms. ${ }^{58}$ As a result, the imbalance of Th1/Th2 may also play some roles in PCa progression.

In summary, we identified the over-expression of HNRNPC mRNA and its correlation with clinicopathologic characteristics and immune infiltration in $\mathrm{PCa}$. We also proved that HNRNPC promotes PCa cell proliferation and metastasis in vitro and predicted its functional mechanisms with the help of bioinformatics analysis. However, no in vivo assays and mechanism validation assays are the main limitations of our study. We will focus on these issues in the future.

\section{Ethical Statement}

This study was conducted in accordance with the Declaration of Helsinki.

\section{Author Contributions}

Shiyu Wang: Conceptualization, Methodology, Investigation, Validation, Formal analysis, Writing - Original Draft, Visualization. Guoxiong $\mathrm{Xu}$ : Conceptualization, Methodology, Writing - Review \& Editing, Resources. Fan Chao: Conceptualization, Writing - Review \& Editing. Cong Zhang: Investigation, Validation. Dunsheng Han: Investigation, Validation. Gang Chen: Conceptualization, Writing - Review \& Editing, Resources, Project administration, Supervision, Funding acquisition. All authors contributed to data analysis, drafting, or revising the article, have agreed on the journal to which the article will be submitted, gave final approval for the version to be published, and agreed to be accountable for all aspects of the work.

\section{Funding}

This work was supported by grants from the Natural Science Foundation of Shanghai (No. 18ZR1405800) and the Project for Key Medical Specialty Construction in Jinshan District (6th Period, Type A) (No. JSZK2019A03) to Gang Chen.

\section{Disclosure}

The authors declare that they have no known competing financial interests or personal relationships that could have appeared to influence the work reported in this paper.

\section{References}

1. Siegel RL, Miller KD, Fuchs HE, Jemal A. Cancer statistics, 2021. CA Cancer J Clin. 2021;71(1):7-33.

2. Teo MY, Rathkopf DE, Kantoff P. Treatment of advanced prostate cancer. Annu Rev Med. 2019;70:479-499.

3. Davies A, Conteduca V, Zoubeidi A, Beltran H. Biological evolution of castration-resistant prostate cancer. Eur Urol Focus. 2019;5 (2):147-154.

4. Furlanis E, Scheiffele P. Regulation of neuronal differentiation, function, and plasticity by alternative splicing. Annu Rev Cell Dev Biol. 2018;34:451-469.

5. Park E, Pan Z, Zhang Z, Lin L, Xing Y. The expanding landscape of alternative splicing variation in human populations. Am J Hum Genet. 2018;102(1):11-26.

6. Urbanski LM, Leclair N, Anczuków O. Alternative-splicing defects in cancer: splicing regulators and their downstream targets, guiding the way to novel cancer therapeutics. Wiley Interdiscip Rev RNA. 2018;9(4):e1476.

7. Martinez-Montiel N, Rosas-Murrieta NH, Anaya Ruiz M, MonjarazGuzman E, Martinez-Contreras R. Alternative splicing as a target for cancer treatment. Int J Mol Sci. 2018;19:2.

8. Geuens T, Bouhy D, Timmerman V. The hnRNP family: insights into their role in health and disease. Hum Genet. 2016;135(8):851-867.

9. Han SP, Tang YH, Smith R. Functional diversity of the hnRNPs: past, present and perspectives. Biochem J. 2010;430(3):379-392.

10. Chaudhury A, Chander P, Howe PH. Heterogeneous nuclear ribonucleoproteins (hnRNPs) in cellular processes: focus on hnRNP E1's multifunctional regulatory roles. RNA. 2010;16(8):1449-1462.

11. Choi YD, Grabowski PJ, Sharp PA, Dreyfuss G. Heterogeneous nuclear ribonucleoproteins: role in RNA splicing. Science. 1986;231 (4745):1534-1539.

12. Merrill BM, Barnett SF, LeStourgeon WM, Williams KR. Primary structure differences between proteins $\mathrm{C} 1$ and C2 of HeLa 40S nuclear ribonucleoprotein particles. Nucleic Acids Res. 1989;17 (21):8441-8449.

13. Wang LC, Chen SH, Shen XL, et al. M6A RNA methylation regulator HNRNPC contributes to tumorigenesis and predicts prognosis in glioblastoma multiforme. Front Oncol. 2020;10:536875.

14. Huang GZ, Wu QQ, Zheng ZN, et al. M6A-related bioinformatics analysis reveals that HNRNPC facilitates progression of OSCC via EMT. Aging. 2020;12(12):11667-11684. 
15. Wu Y, Zhao W, Liu Y, et al. Function of HNRNPC in breast cancer cells by controlling the dsRNA-induced interferon response. EMBO J. 2018;37:23.

16. Fischl H, Neve J, Wang Z, et al. hnRNPC regulates cancer-specific alternative cleavage and polyadenylation profiles. Nucleic Acids Res. 2019;47(14):7580-7591.

17. Park YM, Hwang SJ, Masuda K, et al. Heterogeneous nuclear ribonucleoprotein $\mathrm{C} 1 / \mathrm{C} 2$ controls the metastatic potential of glioblastoma by regulating PDCD4. Mol Cell Biol. 2012;32(20):4237-4244.

18 . Shetty S. Regulation of urokinase receptor mRNA stability by hnRNP C in lung epithelial cells. Mol Cell Biochem. 2005;272(12):107-118.

19. Meng Z, Jackson NL, Choi H, King PH, Emanuel PD, Blume SW. Alterations in RNA-binding activities of IRES-regulatory proteins as a mechanism for physiological variability and pathological dysregulation of IGF-IR translational control in human breast tumor cells. J Cell Physiol. 2008;217(1):172-183.

20. Livak KJ, Schmittgen TD. Analysis of relative gene expression data using real-time quantitative PCR and the 2(-Delta Delta C(T)) method. Methods. 2001;25(4):402-408.

21. Chao F, Song Z, Wang S, et al. Novel circular RNA circSOBP governs amoeboid migration through the regulation of the miR-1413p/MYPT1/p-MLC2 axis in prostate cancer. Clin Transl Med. 2021;11(3):e360.

22. Rhodes DR, Yu J, Shanker K, et al. ONCOMINE: a cancer microarray database and integrated data-mining platform. Neoplasia. 2004;6(1):1-6.

23. Tomczak K, Czerwińska P, Wiznerowicz M. The Cancer Genome Atlas (TCGA): an immeasurable source of knowledge. Contemp Oncol. 2015;19(1a):A68-77.

24. Barrett T, Wilhite SE, Ledoux P, et al. NCBI GEO: archive for functional genomics data sets-update. Nucleic Acids Res. 2013;41 (Database issue):D991-995.

25. Cerami E, Gao J, Dogrusoz U, et al. The cBio cancer genomics portal: an open platform for exploring multidimensional cancer genomics data. Cancer Discov. 2012;2(5):401-404.

26. Abida W, Cyrta J, Heller G, et al. Genomic correlates of clinical outcome in advanced prostate cancer. Proc Natl Acad Sci USA. 2019;116(23):11428-11436.

27. Hänzelmann S, Castelo R, Guinney J. GSVA: gene set variation analysis for microarray and RNA-seq data. BMC Bioinform. 2013;14:7.

28. Barbie DA, Tamayo P, Boehm JS, et al. Systematic RNA interference reveals that oncogenic KRAS-driven cancers require TBK1. Nature. 2009;462(7269):108-112.

29. Team RC. R: A Language and Environment for Statistical Computing [Computer Program]. Vienna, Austria: R Foundation for Statistical Computing; 2019.

30. Bindea G, Mlecnik B, Tosolini M, et al. Spatiotemporal dynamics of intratumoral immune cells reveal the immune landscape in human cancer. Immunity. 2013;39(4):782-795.

31. Zhou Y, Zhou B, Pache L, et al. Metascape provides a biologist-oriented resource for the analysis of systems-level datasets. Nat Commun. 2019;10(1):1523.

32. Shishkin SS, Kovalev LI, Pashintseva NV, Kovaleva MA, Lisitskaya K. Heterogeneous nuclear ribonucleoproteins involved in the functioning of telomeres in malignant cells. Int $\mathrm{J} \mathrm{Mol} \mathrm{Sci}$. 2019;20:3.

33. Yang Y, Chen Q, Piao HY, et al. HNRNPAB-regulated IncRNA-ELF209 inhibits the malignancy of hepatocellular carcinoma. Int $J$ Cancer. 2020;146(1):169-180.

34. Fei T, Chen Y, Xiao T, et al. Genome-wide CRISPR screen identifies HNRNPL as a prostate cancer dependency regulating RNA splicing. Proc Natl Acad Sci USA. 2017;114(26):E5207-e5215.
35. Errichelli L, Dini Modigliani S, Laneve P, et al. FUS affects circular RNA expression in murine embryonic stem cell-derived motor neurons. Nat Commun. 2017;8:14741.

36. Liu X, Zhou Y, Lou Y, Zhong H. Knockdown of HNRNPA1 inhibits lung adenocarcinoma cell proliferation through cell cycle arrest at G0/G1 phase. Gene. 2016;576(2 Pt 2):791-797.

37. Loh TJ, Moon H, Cho S, et al. CD44 alternative splicing and hnRNP A1 expression are associated with the metastasis of breast cancer. Oncol Rep. 2015;34(3):1231-1238.

38. Li H, Guo L, Huang A, et al. Nanoparticle-conjugated aptamer targeting hnRNP A2/B1 can recognize multiple tumor cells and inhibit their proliferation. Biomaterials. 2015;63:168-176.

39. Balasubramani M, Day BW, Schoen RE, Getzenberg RH. Altered expression and localization of creatine kinase $\mathrm{B}$, heterogeneous nuclear ribonucleoprotein $\mathrm{F}$, and high mobility group box 1 protein in the nuclear matrix associated with colon cancer. Cancer Res. 2006;66(2):763-769.

40. Li F, Zhao H, Su M, et al. HnRNP-F regulates EMT in bladder cancer by mediating the stabilization of Snaill mRNA by binding to its $3^{\prime}$ UTR. EBioMedicine. 2019;45:208-219.

41. Li F, Xie W, Fang Y, et al. HnRNP-F promotes the proliferation of bladder cancer cells mediated by PI3K/AKT/FOXO1. J Cancer. 2021;12(1):281-291.

42. Montero-Conde C, Graña-Castro O, Martín-Serrano G, et al. HsamiR-139-5p is a prognostic thyroid cancer marker involved in HNRNPF-mediated alternative splicing. Int $J$ Cancer. 2020;146 (2):521-530

43. Dlamini Z, Mokoena F, Hull R. Abnormalities in alternative splicing in diabetes: therapeutic targets. J Mol Endocrinol. 2017;59(2):R93-r107.

44. Narayanan SP, Singh S, Shukla S. A saga of cancer epigenetics: linking epigenetics to alternative splicing. Biochem J. 2017;474 (6):885-896

45. Bhadra M, Howell P, Dutta S, Heintz C, Mair WB. Alternative splicing in aging and longevity. Hum Genet. 2020;139(3):357-369.

46. Bowler E, Oltean S. Alternative splicing in angiogenesis. Int $\mathrm{J} \mathrm{Mol}$ Sci. 2019;20:9.

47. Song H, Wang L, Chen D, Li F. The function of Pre-mRNA alternative splicing in mammal spermatogenesis. Int J Biol Sci. 2020;16(1):38-48.

48. Lara-Pezzi E, Gómez-Salinero J, Gatto A, García-Pavía P. The alternative heart: impact of alternative splicing in heart disease. J Cardiovasc Transl Res. 2013;6(6):945-955.

49. Dredge BK, Polydorides AD, Darnell RB. The splice of life: alternative splicing and neurological disease. Nat Rev Neurosci. 2001;2 (1):43-50.

50. Farina AR, Cappabianca L, Sebastiano M, Zelli V, Guadagni S, Mackay AR. Hypoxia-induced alternative splicing: the 11th hallmark of cancer. J Exp Clin Cancer Res. 2020;39(1):110.

51. Shimasaki N, Jain A, Campana D. NK cells for cancer immunotherapy. Nat Rev Drug Discov. 2020;19(3):200-218.

52. Morvan MG, Lanier LL. NK cells and cancer: you can teach innate cells new tricks. Nat Rev Cancer. 2016;16(1):7-19.

53. Orange JS. Natural killer cell deficiency. J Allergy Clin Immunol. 2013;132(3):515-525.

54. Reizis B. Plasmacytoid dendritic cells: development, regulation, and function. Immunity. 2019;50(1):37-50.

55. Mitchell D, Chintala S, Dey M. Plasmacytoid dendritic cell in immunity and cancer. $J$ Neuroimmunol. 2018;322:63-73.

56. Greene TT, Jo YR, Zuniga EI. Infection and cancer suppress pDC derived IFN-I. Curr Opin Immunol. 2020;66:114-122.

57. Sallusto F, Geginat J, Lanzavecchia A. Central memory and effector memory T cell subsets: function, generation, and maintenance. Annu Rev Immunol. 2004;22:745-763.

58. Kidd P. Th1/Th2 balance: the hypothesis, its limitations, and implications for health and disease. Altern Med Rev. 2003;8(3):223-246. 


\section{Publish your work in this journal}

Cancer Management and Research is an international, peer-reviewed The manuscript management system is completely online and includes open access journal focusing on cancer research and the optimal use of a very quick and fair peer-review system, which is all easy to use. preventative and integrated treatment interventions to achieve improved Visit http://www.dovepress.com/testimonials.php to read real quotes outcomes, enhanced survival and quality of life for the cancer patient.

from published authors. 